\title{
Spectrum of imaging and characteristics for liver tumors treated with irreversible electroporation
}

\author{
Robert E. Neal II ${ }^{1 *}$, Wa Cheung ${ }^{1 *}$, Helen Kavnoudias ${ }^{1}$, Kenneth R. Thomson ${ }^{2}$ \\ ${ }^{1}$ Department of Radiology, The Alfred Hospital, Melbourne, Australia \\ ${ }^{2}$ Departments of Radiology and Surgery, The Alfred Hospital, Melbourne, Australia \\ Email: robert.neal@alfred.org.au
}

Received 6 October 2012; revised 12 November 2012; accepted 22 November 2012

\begin{abstract}
Therapeutic irreversible electroporation (IRE) is a relatively new technique for targeted tumor ablation. Needle electrodes are placed into or around the targeted region to deliver a series of brief electric pulses that disrupt cell membrane integrity, killing cells in a non-thermal manner that does not affect the extracellular matrix or sensitive structures such as major vasculature and bile ducts; making IRE an advantageous technique, especially for tumors that are unresectable or ineligible for thermal ablation. Here, we present on the imaging findings from IRE liver tumor treatments from ultrasound, computed tomography, magnetic resonance, and positron emission tomography. Imaging aids planning and implementing treatments by visualizing the targeted volume and guiding electrode placement. Immediate changes to the affected IRE region may be observed to verify complete ablation of the tumor with margin during the procedure, and permits follow-up evaluation of clinical outcome. In particular, we present tumor treatments in regions adjacent to sensitive structures that contraindicate thermal therapies.
\end{abstract}

Keywords: IRE, Pictorial Review; Non-Thermal Focal Ablation; Minimally Invasive Surgery; Medical Imaging

\section{INTRODUCTION}

Surgical resection is regarded as the standard curative treatment option for primary and metastatic liver tumors. Although screening at-risk patients for hepatocellular carcinoma is now a common practice and allows detection tumors at an early stage, many tumors remain ineligible for resection at the time of diagnosis. The majority of patients diagnosed with hepatocellular carcinoma have unresectable disease due to advanced liver Cirrhosis or multicentricity [1,2]. Focally-targeted tech-

*Both authors contributed equally to this work. niques for regional destruction of tissue are beginning to be used as an alternative to resection in the treatment of tumors, reducing invasiveness and exhibiting utility in unresectable cases [3].

Common focal ablation therapies achieve cell death through chemical approaches, such as percutaneous ethanol ablation and transarterial chemoembolization; as well as targeted thermal manipulations, such as radiofrequency ablation (RFA), cryoablation, laser interstitial thermal therapy, or high-intensity focused ultrasound. RFA has shown particular utility in the treatment of hepatic tumors, attaining 2-year local recurrence-free survival rates of $96 \%$ in small hepatocellular carcinomas, comparable to partial hepatectomy [4]. While producing satisfactory results in most cases, there remain tumors ineligible for treatment by existing focal ablation treatments due to the risk of injury for tumors near sensitive structures, such as the common bile duct and porta hepatis [5]. In addition, blood perfusion-induced "heat sink" effects mitigate the lethality of thermal therapies adjacent to major blood vessels [3].

Focally-targeted cancer treatment success supports the emergence of additional ablation techniques to treat liver tumors, including irreversible electroporation (IRE) [6]. IRE treatments use a series of approximately 80 to 100 brief ( 100 $\mu$ s long) but intense ( 1 - $3 \mathrm{kV}$ ) square-wave electric pulses delivered directly to the tissue by small needle electrodes placed into or around the targeted region. The pulses alter the transmembrane potential of the cells, leading to the creation of irrecoverable nano-scale defects that result in death of the cells in the targeted region [7]. Typical pulses are delivered between pairs of parallel electrodes separated by 0.5 to $2.5 \mathrm{~cm}$ with 1 to 3 $\mathrm{cm}$ exposure lengths. The needle electrodes are to be inserted at the margin and/or inside the tumor, depending on the size and configuration of the lesion, in order to achieve complete ablation of the tumor and a 5 to $10 \mathrm{~mm}$ margin of surrounding normal appearing liver tissue.

The resulting non-thermal mode of cell death does not affect the supporting extracellular matrix, sparing sensi- 
tive structures such as bile ducts, neurovascular bundles, and major vasculature [8-10]. This permits IRE treatment for tumors deemed unresectable or ineligible for other focal ablation techniques. IRE causes rapid lesion creation with cellular-scale demarcation between treated and unaffected cells, and is unaffected by blood perfusion heat sink $[8,11]$. Furthermore, treatments may be predicted with numerical models for treatment planning, and effects may be monitored in real-time with imaging [11, 12]. IRE has been shown to successfully treat a variety of cancers in experimental models and spontaneous tumors [13-16]; with a human safety study attaining complete tumor remission in 46 of 69 tumors (67\%) ineligible or unresponsive to conventional therapies [10]. These studies demonstrate IREs ability to ablate without damaging sensitive anatomical structures or losing efficacy near blood vessels due to "heat sink" effects.

Experimental animal studies have shown the ability to observe the effects of IRE in real-time with ultrasound $[9,17]$, with a strong association shown between ultrasound changes and the macroscopic and histologic ablation realms [18]. This report presents some of the radiological findings for IRE therapies performed on patient liver tumors. Various medical imaging modality responses to IRE are described as well as presentation of images demonstrating IREs ability to be used clinically in proximity to various sensitive structures that contraindicate other therapeutic options for tumors in the liver.

\section{ULTRASOUND}

Ultrasound is a suitable imaging modality to guide IRE procedural needle placement and to monitor the effects of treatments in real-time. Tumors in liver usually present as solid masses with same reflectivity surrounded by a rim of lower reflectivity (hypoechogenic) or completely hypoechogenic region, providing easy visualization of the targeted mass and enabling observation of needle electrode placement relative to the target for accurate ablation margins (Figure 1). In cirrhotic livers and obese patients, a hepatocellular carcinoma may be difficult to differentiate with ultrasound.

There can be multiple responses to IRE ablation in ultrasound due to effects from the electric pulses on the tissue. As noted in $[9,18]$, electroporated regions become hypoechogenic (Figure 2). This initial response occurs within 1 - 2 minutes of pulses delivery, which experimentally evolves 90 - 120 minutes after ablation into a hyperechogenic (higher reflectivity or brighter than surrounding liver) rim with an isoechogenic (same reflectivity as surrounding liver) central region. The experimental rim was shown to best correlate with the ablated volume, while the immediate effects on ultrasound provide a good initial approximation, but may overestimate

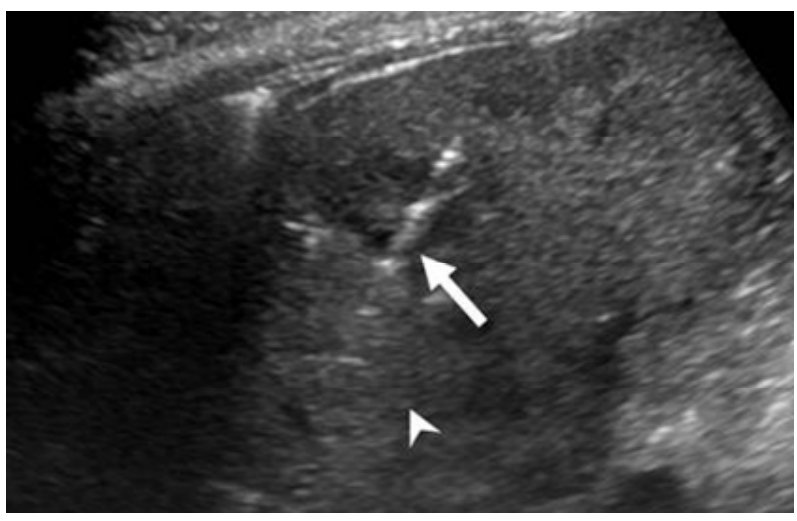

Figure 1. Ultrasound of liver demonstrating presence of a single IRE electrode (arrow) for guiding placement into the targeted region (arrowhead).

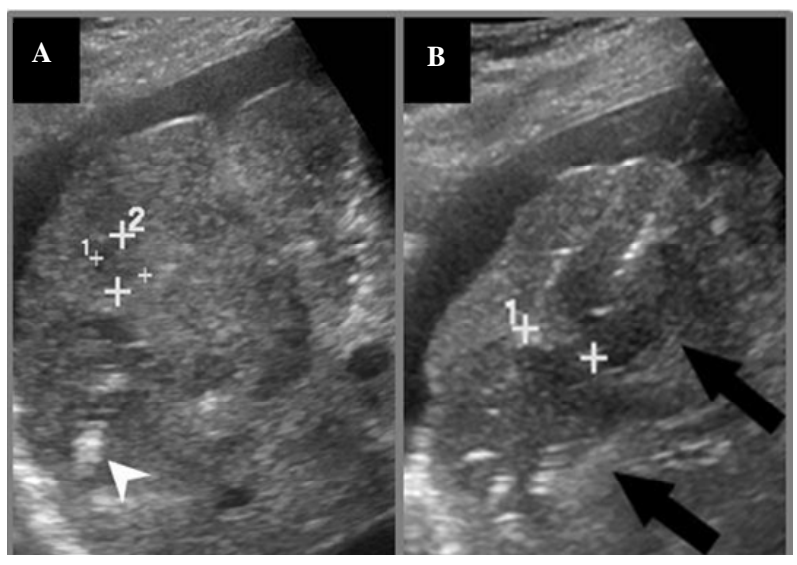

Figure 2. Ultrasound images of targeted mass during IRE. (A) Cursors denote remaining tumor margin after a prior ablation in the deeper aspect of the tumor, showing linear microbubbles within a hypoechogenic ablation zone (arrow- head); (B) Second ablation performed within superficial region of targeted mass immediately post-IRE showing 2 electrodes (crosshairs) within a hypoechoic ablation zone (black arrows). The cursor is used to measure the distance between the needle electrodes, which is critical for ablation success.

the treated volume by 3 - $4 \mathrm{~mm}$ [18], so care should be taken to ensure adequate margins beyond the targeted volume when using the immediate response to confirm complete tumor ablation.

In addition to ablated regions becoming hypoechogenic on ultrasound, the electric pulses will cause hydrolytic effects in the tissue, resulting in the formation of microbubbles. Depending on the pulsing protocol used, these microbubbles may cover the entire ablation zone (Figure 3).

Where this modality does not require radiation or strong magnets that may affect IRE components or equipment, ultrasound is a useful tool to aid IRE procedures when a clear window to the tumor exists and the targeted region can be visualized. In addition, contrastenhanced ultrasound via microbubbles may provide fur- 


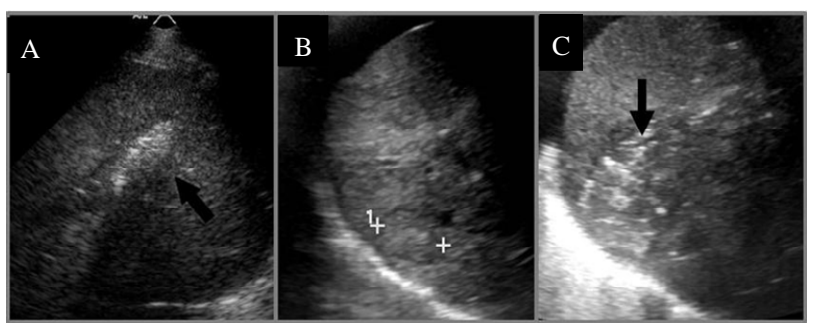

Figure 3. (A) Ultrasound image immediately post-IRE showing microbubbles within the entire ablation zone (ar- row); (B) Ultrasound of liver tumor prior to; and (C) 5 minutes post-IRE ablation showing the ablation zone and gas formation (arrow) throughout the lesion. The cursors in (B) are used to measure the distance between the margins of the tumor for protocol planning.

ther benefit to guiding and monitoring treatments by improving depiction of tumor vascularity while inducing less toxicity than contrast for MRI or CT, though such a benefit to procedures and therapeutic outcomes remains to be determined.

\section{COMPUTED TOMOGRAPHY (CT)}

Treatment planning, electrode guidance, immediate evaluation, and further follow-up may be performed with computed tomography (CT). Contrast-enhanced CT enables visualization of many liver tumors immediately prior to treatment to determine the targeted volume (Figure 4(A)). In addition, fluoroscopic and standard CT protocols may be used to facilitate and check electrode placement prior to electric pulse delivery (Figure 4(B)). Following treatment, CT may be repeated to ensure the realm of ablation encompasses the originally targeted volume with a good margin.

The radiologic effects from IRE are similar to radiofrequency ablation in computed tomography (CT). Typical CT findings immediately after IRE show an ablation zone centered around the electrodes that does not enhance post-contrast (Figure 4(C)). There is, however, an immediate transient peripheral rim of contrast enhancement indicating hyperemia (Figure 5(A)) [19]. Gas formation resulting from the electric pulses can also be noted on CT (Figure 5(B)). Similar to RFA treatments, care should be taken when evaluating immediate postIRE outcome to ensure that this hyperemic rim is not confused with regions of residual tumor, which demonstrates focal and irregular peripheral enhancement [20]. Of note, progressive follow-up imaging of IRE results with CT show that IRE ablation lesions resolve faster than comparable RFA ablations (Figure 6).

\section{MAGNETIC RESONANCE IMAGING}

Primary or metastatic liver tumors in the liver are usually well demonstrated on CT. Most primary tumors enhance prior to ablation. Because the ablation zone does not

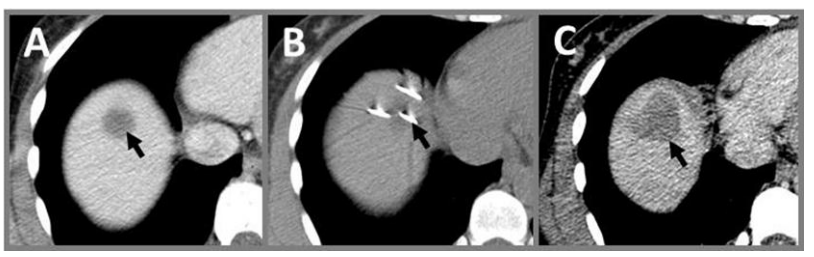

Figure 4. Use of CT in IRE procedures. (A) Contrast- enhanced image prior to IRE showing targeted volume (arrow); (B) CT image of electrodes (arrow) placed in targeted volume prior to pulse delivery; (C) Follow-up contrast en- hanced CT immediately post-IRE showing a hypointense region of ablation (arrow) that completely covers the origin- nally targeted volume in (A).

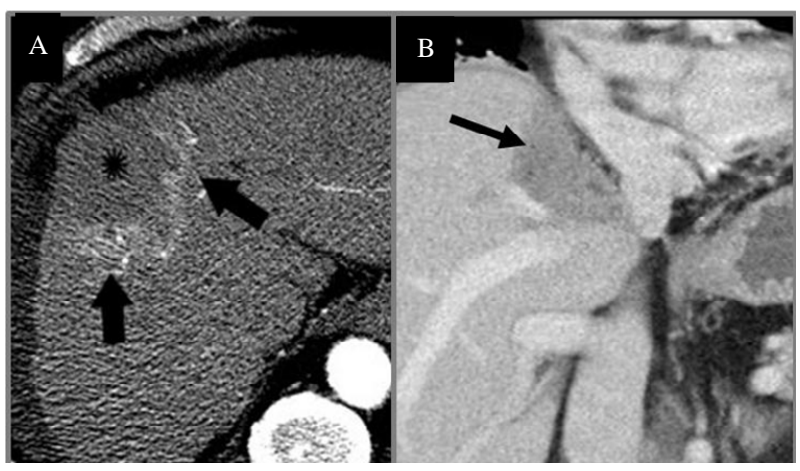

Figure 5. CTs immediately post-IRE. (A) CT of Abdomen immediately post-IRE shows necrotic tumor (star) with peripheral hyperemia (arrows); (B) Coronal CT of lesion adjacent to portal vein and inferior vena cava immediately post-IRE, showing gas formation (arrows) caused by elec- trolysis.

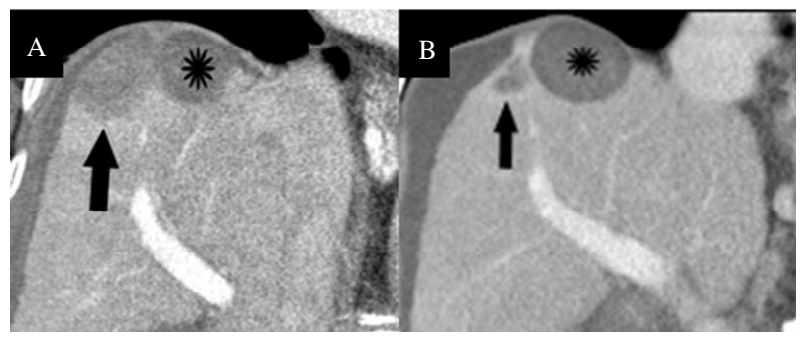

Figure 6. Progression of IRE lesion compared to RFA. (A) Contrast-enhanced coronal CT image obtained immediately post-IRE showing IRE-induced necrotic tumor in the dome of the liver (arrow), which is similar in appearance to the RFA-induced necrosis of the other lesion (star), which received treatment 8 months prior; (B) Repeat CT imaging performed 1 year post-IRE, which shows a more rapid re- duction in size of the IRE ablation zone (arrow) compared to the RFA ablation zone (star).

enhance, ablation zones relative to residual tumor may be easily assessed. However, metastatic tumors enhance poorly on CT compared to normal liver, and may pose difficulty in differentiating residual tumor cells from the ablation zones. MRI provides a useful tool for evaluating IRE procedures without requiring exposure to radiation and most metastatic tumors appear hyperintense or brighter than the surrounding liver on T2- 
weighted images. IRE post-treatment ablations demonstrate similar appearances to RFA on MRI. T2-weighted MRI shows a hyper-intense reactive rim surrounding the hypo-intense necrotic center of the ablated region (Figure 7(A)). T1-weighted MRI is less useful as it demonstrates heterogeneous hypointense or mixed signal in the ablation region, including the reactive rim (Figures 7(B) and $(\mathbf{C})$ ). Similar to CT, the necrotic scar does not enhance, while the reactive rim will enhance on the post contrast T1-weighted image.

\section{POSITRON EMISSION TOMOGRAPHY}

Positron emission tomography (PET) scan, performed with fluorine $18\left({ }^{18} \mathrm{~F}\right)$ fluorodeoxyglucose (FDG), is one of the functional imaging modalities used to demonstrate glucose metabolism in living human tissues. FDG, an analog of glucose, is transported into cells for glucose metabolism. It becomes metabolically trapped and accumulates within the cells at a rate proportional to glucose utilization. FDG preferentially accumulates in cancers because of their increased glucose metabolism. However, increased glucose metabolism is not specific to cancer. FDG uptake can be seen in many noncancerous disorders including inflammation or infection. PET scans of IRE ablations show a dynamic response to the IRE ablation. At three days following IRE therapy, an FDG avid peripheral zone surrounding the ablated region is present (Figure 8(B)). However, at one month following IRE, this peripheral increase in FDG uptake is no longer present (Figure 8(C)). The initial increase in uptake at the periphery of the IRE region may be due to an initial inflammatory response increasing metabolic activity at the targeted region as the cellular debris is removed from the targeted site. Therefore, accurate assessment of IRE treatment outcome with PET should be performed after this initial response has resolved.

\section{IRREVERSIBLE \\ ELECTROPORATION FOR LIVER TUMORS NEAR SENSITIVE STRUCTURES}

Previous studies have demonstrated IREs ability to ablate volumes of tissue without destroying nearby delicate structures, including neurovascular bundles or major blood vessels $[8,10]$. Here, we present liver tumors treated with IRE that are adjacent to sensitive anatomical features (Figures 9-13). These tumors were unsuitable for surgical resection and/or thermal ablation. Reports of thermal ablations in these locations have included complications such as cardiac tamponade, bile duct stricture, and perforation of bowel and gallbladder.

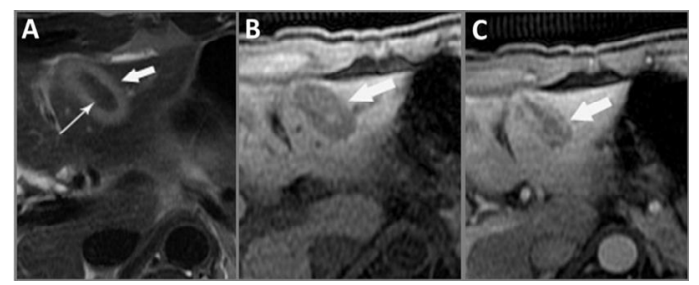

Figure 7. MRI findings from IRE. (A) T2-weighted axial MRI of the liver 2 days post-IRE shows a hyper-intense reactive rim (bold arrow) surrounding the ablated region with a hypo-intense necrotic center (thin arrow); (B) T1- weighted axial MRI two days post-IRE demonstrates mixed signal intensity (arrow) comprised of a hypo-intense ablated zone containing a hyper-intense central signal; (C) Post- contrast enhanced hepatic arterial phase axial T1-weighted MRI shows a non-enhancing linear ablation zone and hyper-enhancing peripheral rim (arrow), corresponding to the hyperemic rim in the T2-weighted image (A).

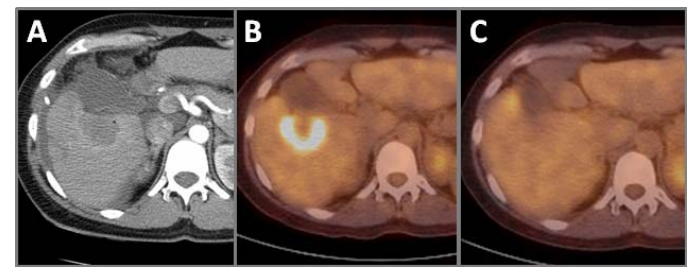

Figure 8. Positron emission tomography of IRE ablation. (A) Contrast-enhanced CT immediately postIRE showing ablation zone. (B) Peripheral zone of IRE-ablated region shows increased FDG uptake three days post-IRE. (C) At one month post-IRE, PET does not show increased FDG uptake within the ablated zone or at the hyperemic reactive rim.

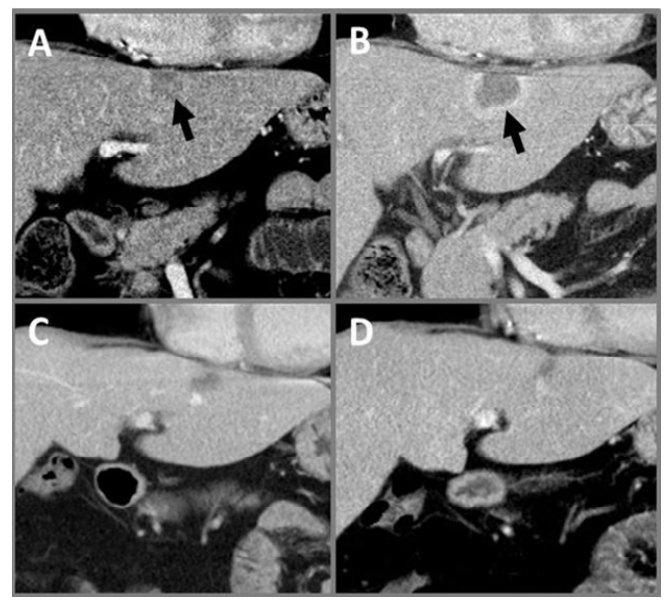

Figure 9. Progressive CTs of IRE Liver Tumor. Coronal CT abdomen images (A) prior to treatment with targeted volume (arrow) and (B) immediately post- IRE showing no contrast enhancement and hypere- mic rim. Continued follow-up shows lesion resolu- tion at (C) one month and (D) nine months post-IRE showing continued scar retraction. All post-IRE im- ages show no damage to surrounding structures, including diaphragm and pericardium. 


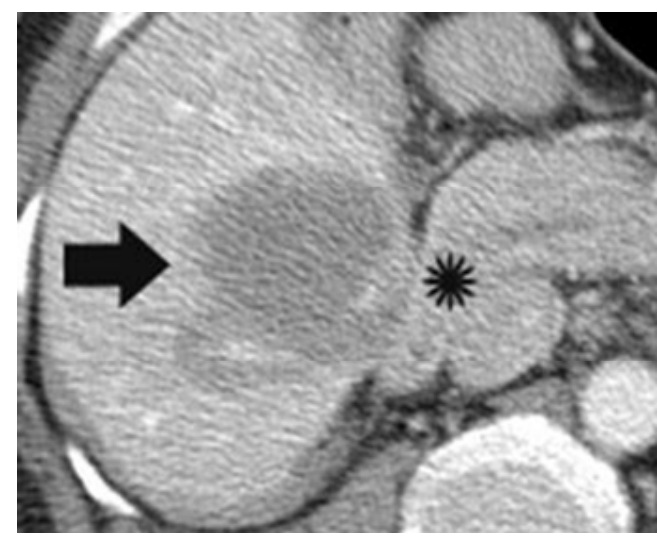

Figure 10. Axial portal venous phase contrast CT of abdo- men immediately post-IRE demonstrates treatment of hea- ptic tumor (bold arrow) abutting the second part of the duodenum (star). There was no evidence of duodenal per- foration on follow-up.

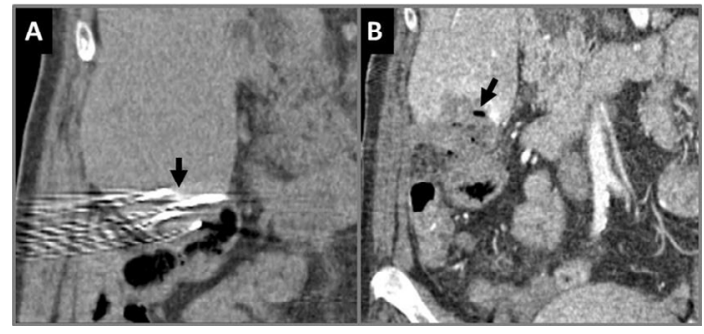

Figure 11. Coronal CT showing of IRE procedure at in- ferior aspect of liver. (A) Needle placements (arrow) are shown very close to bowel prior to pulse delivery; (B) Im- mediately post-IRE showing gas formation (arrow). The bowel wall next to the ablation zone appears thickened but without perforation.

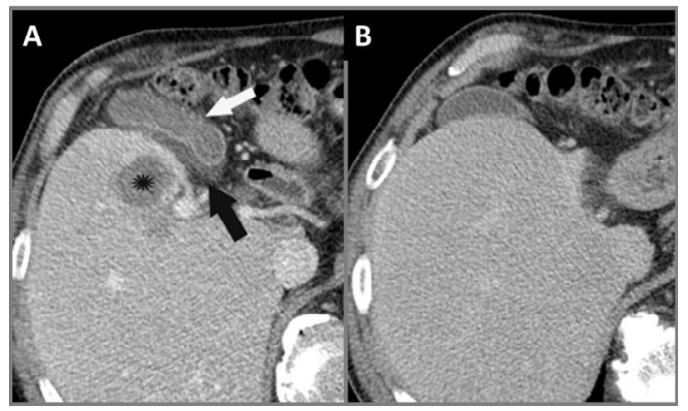

Figure 12. Axial CT of abdomen (A) 1 day and (B) 3 months post-IRE. Panel (A) demonstrates hepatic tumor necrosis (star) with associated gall bladder thickening (white arrow) and edema with mucosal enhancement (black arrow). Panel (B) shows that the gallbladder remained intact and returns to normal appearance on follow-up CT.

\section{CONCLUSION}

Irreversible electroporation is a new and promising minimally invasive targeted ablation technique for the treatment of liver tumors. Its unique mode of cell destruction

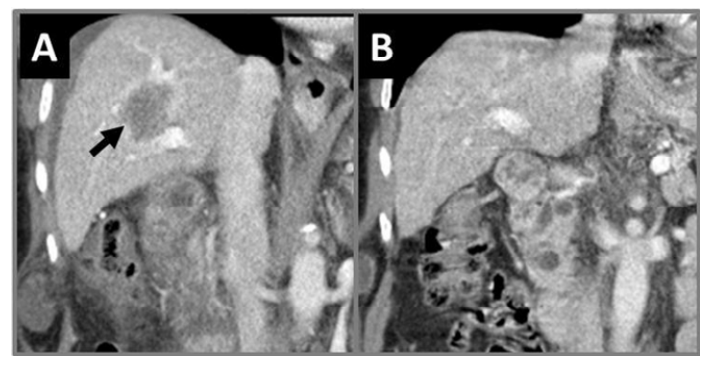

Figure 13. Coronal CT of abdomen with IRE-treated liver tumor. (A) Immediately post-IRE therapy shows treated liver tumor (arrow) encasing the portal vein and possibly the intrahepatic bile ducts. (B) 30 month post-IRE shows no residual tumor with patent portal vein and no bile duct stricture.

spares sensitive structures and is unaffected by blood perfusion thermal dissipative effects, making it useful for tumors unresectable or untreatable by other methods. Tumor and liver radiologic response to IRE is similar to that of radiofrequency ablation. Ultrasound provides a method to guide electrode placement and monitor effects to the tissue in real-time for ensuring complete coverage of the targeted region, producing hypoechoic regions or gas formation within the affected tissue. Computed tomography can be used to check electrode placement prior to electric pulse delivery, and shows ablations typically comprised of a non-enhancing ablation region with peripheral hyperemia immediately post-IRE, with lesion resolution faster than that found from radiofrequency ablation. Varied lesion response is shown on MRI, with T2weighting showing a hyperintense reactive rim surrounding the hypointense necrotic center of the ablation and a heterogeneous hypointense or mixed signal of the ablation on T1-weighted images. Positron emission tomography shows increased FDG uptake several days following IRE, which resolves by one month. IRE is also shown to be safe and effective in treating tumors contraindicated for resection or thermal ablation due to their proximity to sensitive structures.

\section{ACKNOWLEDGEMENTS}

This work supported by the Flack Trustees and Whitaker International Programs. The authors thank Paul Marovic for his assistance in this composition.

\section{REFERENCES}

[1] Schafer, D.F. and Sorrell, M.F. (1999) Hepatocellular carcinoma. Lancet, 353, 1253-1257. doi:10.1016/S0140-6736(98)09148-X

[2] T. Tsuzuki, et al. (1990) Hepatic resection for hepatocellular carcinoma. Surgery, 107, 511-520.

[3] Ahmed, M., Brace, C.L., Lee, F.T. and Goldberg, S.N. 
(2011) Principles of and advances in percutaneous ablation. Radiology, 258, 351-369.

doi:10.1148/radiol.10081634

[4] Lencioni, R.A., et al. (2003) Small hepatocellular carcinoma in cirrhosis: Randomized comparison of radio-frequency thermal ablation versus percutaneous ethanol injection. Radiology, 228, 235-240. doi:10.1148/radiol.2281020718

[5] Lencioni, R. (2010) Loco-regional treatment of hepatocellular carcinoma. Hepatology, 52, 762-773. doi:10.1002/hep.23725

[6] Davalos, R., Mir, L. and Rubinsky, B. (2005) Tissue ablation with irreversible electroporation. Annals of Biomedical Engineering, 33, 223-231.

[7] Weaver, J.C. (1993) Electroporation: A general phenomenon for manipulating cells and tissue. Journal of Cellular Biochemistry, 51, 426-435.

[8] Onik, G., Mikus, P. and Rubinsky, B. (2007) Irreversible electroporation: Implications for prostate ablation. Technology in Cancer Research and Treatment, 6, 295-300.

[9] Lee, E.W., Loh, C.T. and Kee, S.T. (2007) Imaging guided percutaneous irreversible electroporation: ultrasound and immunohistological correlation. Technology in Cancer Research and Treatment, 6, 287-293.

[10] Thomson, K.R. et al. (2011) Investigation of the safety of irreversible electroporation in humans. Journal of Vascular and Interventional Radiology, 22, 611-621. doi:10.1016/j.jvir.2010.12.014

[11] Edd, J.F., Horowitz, L., Davalos, R.V., Mir, L.M. and Rubinsky, B. (2006) In vivo results of a new focal tissue ablation technique: Irreversible electroporation. IEEE Transactions on Biomedical Engineering, 53, 1409-1415. doi:10.1109/TBME.2006.873745

[12] Miklavcic, D., Semrov, D., Mekid, H. and Mir, L.M. (2000) A validated model of in vivo electric field distribution in tissues for electrochemotherapy and for DNA electrotransfer for gene therapy. Biochimica et Biophy- sica Acta, 1523, 73-83. doi:10.1016/S0304-4165(00)00101-X

[13] B. Al-Sakere, et al. (2007) Tumor ablation with irreversible electroporation. PLoS One, 2, e1135. doi:10.1371/journal.pone.0001135

[14] Neal, R.E., et al. (2011) Successful treatment of a large soft tissue sarcoma with irreversible electroporation. Journal of Clinical Oncology, 29, e372-e377. doi:10.1200/JCO.2010.33.0902

[15] Neal, R. E., et al. (2010) Treatment of breast cancer through the application of irreversible electroporation using a novel minimally invasive single needle electrode. Breast Cancer Research and Treatment, 123, 295-301. Aug. doi:10.1007/s10549-010-0803-5

[16] Garcia, P.A., et al. (2011) Non-thermal irreversible electroporation (N-TIRE) and adjuvant fractionated radiotherapeutic multimodal therapy for intracranial malignant glioma in a canine patient. Technology in Cancer Research and Treatment, 10, 73-83.

[17] Rubinsky, B., Onik, G. and Mikus, P. (2007) Irreversible electroporation: A new ablation modality: Clinical implications. Technology in Cancer Research and Treatment, 6, 37-48.

[18] Appelbaum, L., Ben-David, E., Sosna, J., Nissenbaum, Y. and Goldberg, S.N. (2012) US findings after irreversible electroporation ablation: Radiologic-pathologic correlation. Radiology, 262, 117-125. doi:10.1148/radiol.11110475

[19] Kim, Y.S., et al. (2011) Coagulation necrosis induced by radiofrequency ablation in the liver: Histopathologic and radiologic review of usual to extremely rare changes. $\mathrm{Ra}$ diographics, 31, 377-390.doi:10.1148/rg.312105056

[20] Kim, S.K., et al. (2003) Hepatocellular carcinoma treated with radio-frequency ablation: Spectrum of imaging findings. Radiographics, 23, 107-121. doi:10.1148/rg.231025055 